\title{
Acute and Mid-Term Results after Pulmonary Veins Isolation Using a Novel Circular Irrigated Multielectrode Mapping and Ablation Catheter (nMARQ ${ }^{\text {TM}}$ )
}

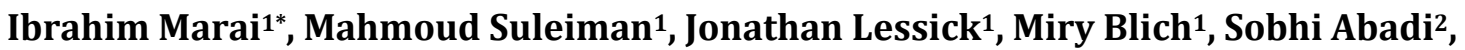 \\ Monther Boulos ${ }^{1}$
}
${ }^{1}$ Division of Pacing and Electrophysiology, Rambam Health Care Campus and Bruce Rappaport Faculty of Medicine, Technion-Israel Institute of Technology, Haifa, Israel
${ }^{2}$ Department of Diagnostic Imaging, Rambam Health Care Campus and Bruce Rappaport Faculty of Medicine, Technion-Israel Institute of Technology, Haifa, Israel

Email: ^ibrahim_marai@yahoo.com

How to cite this paper: Marai, I., Suleiman, M., Lessick, J., Blich, M., Abadi, S. and Boulos, M. (2016) Acute and Mid-Term Results after Pulmonary Veins Isolation Using a Novel Circular Irrigated Multielectrode Mapping and Ablation Catheter (nMARQ ${ }^{\mathrm{TM}}$ ). World Journal of Cardiovascular Diseases, 6, 477-488.

http://dx.doi.org/10.4236/wjcd.2016.612051

Received: April 5, 2016

Accepted: December 27, 2016

Published: December 30, 2016

Copyright $\odot 2016$ by authors and Scientific Research Publishing Inc. This work is licensed under the Creative Commons Attribution International License (CC BY 4.0).

http://creativecommons.org/licenses/by/4.0/

(c) (i) Open Access

\section{Abstract}

Background: Pulmonary vein isolation (PVI) is the cornerstone of atrial fibrillation (AF) ablation in both paroxysmal and persistent AF. However, this procedure is still challenging and time consuming. Objective: The aim of this study was to present our approach for PVI using a novel circular irrigated multielectrode mapping and ablation catheter (nMARQ ${ }^{\mathrm{TM}}$ ) and to present acute and mid-term results. Methods: The study included 31 consecutive patients with symptomatic AF (4 had persistent and 27 had paroxysmal AF) who underwent PVI using the nMARQ ${ }^{\mathrm{TM}}$ catheter. Circular ablation was guided by CT image integrated into fast anatomical map and by intra cardiac echo. Isolation of pulmonary veins was identified using the nMARQ ${ }^{\mathrm{TM}}$ catheter if it was possible to advance it into the veins, otherwise Lasso catheter was used. Patients were followed up to 20 months. Results: PVI was achieved in 119 (98\%) out of 121 pulmonary veins identified, and final PVI was obtained in 30 (97\%) out of 31 patients. Lasso catheter was needed for PVI confirmation in 16 (52\%) patients. Touch up ablation using standard catheter guided by Lasso catheter was needed in $4(13 \%)$ patients. Pericardial tamponade occurred in 1 patient who was treated with pericardiocentesis. No other major complications were detected. During follow-up (mean $15.9 \pm 3.6$ months, range 9 - 20 months), 4 (13\%) patients had recurrence of atrial tachyarrhythmia. Conclusion: PVI using the novel nMARQ ${ }^{\mathrm{TM}}$ catheter is safe with good acute and mid-term efficacy. Long term follow up trials are needed. 


\section{Keywords}

Atrial Fibrillation, Catheter Ablation, Image Integration, nMARQ, Lasso

\section{Introduction}

In treatment of symptomatic and drug refractory atrial fibrillation (AF), catheter-based pulmonary vein isolation (PVI) has been established as a standard procedure by using a single-tip ablation catheter for creating linear lesion surrounding ipsilateral pulmonary veins (PVs) [1]. However, this procedure is still time consuming and requires experienced operators and centers. In addition, repeat procedures are required in a significant number of cases and recurrent PV conduction is responsible for most ablation failures in paroxysmal $\mathrm{AF}[2]$.

The so-called single-shot devices such as cryoballoon and pulmonary vein ablation catheter (PVAC) aimed at the creation of circumferential lesions by only a few applications have been developed and are in clinical use in the recent years [3] [4]. Recently, a novel irrigated multi electrode mapping and ablation catheter $\left(\mathrm{nMARQ}^{\mathrm{TM}}\right.$ catheter, Biosense Webster, Diamond Bar, CA) was introduced for PVI. The nMARQ ${ }^{\mathrm{TM}}$ catheter is an 8.4-F decapolar mapping and radiofrequency ( $\mathrm{RF}$ ) ablation catheter with an adjustable circular array of a diameter between 20 and $35 \mathrm{~mm}$ [5]. Platinum electrodes are $3 \mathrm{~mm}$ long, with a spacing of $4 \mathrm{~mm}$. Each of the electrodes possesses a thermocouple and holes for irrigation. This catheter has the capability of combining both 3-dimensional (3D) electroanatomic mapping and multipolar RF ablation through open irrigation design.

Preliminary reports on PVI with nMARQ showed a good acute success. The nMARQ ablation system provides both short procedure and fluoroscopy times compared with other existing ablation systems [5]. However, long term follow up is lacking. The aim of this study is to present our approach of PVI using this new technology and midterm clinical follow-up up to 20 months.

\section{Methods}

We summarized our initial experience of PVI using nMARQ ${ }^{\mathrm{TM}}$ circular catheter guided by CT image integration into an electro anatomic map and intracardiac echo (ICE) during 2014. All patients gave written informed consent before the procedure according to local institutional guides.

The procedure was done during deep sedation with fentanyl and midazolam. Single trans-septal puncture was done with guidance of ICE, and steerable sheath was introduced to left atrium (LA). The nMARQ ${ }^{\mathrm{TM}}$ catheter was advanced to LA via the steerable sheath. Intravenous unfractionated heparin (UFH; $100 \mathrm{IU} / \mathrm{kg}$ ) was administered after trans-septal puncture. The activated clotting time (ACT) was determined every $30 \mathrm{mi}$ nutes and additional boluses of UFH were administered aiming to maintain ACT $>320$ seconds throughout the procedure. Initially, fast anatomic map (FAM) of LA and LA/ PVs junctions was performed. The ostium and veno-atrial junction of each PV was 
identified by fluoroscopic guidance, intra cardiac electrogram from the nMARQ ${ }^{\mathrm{TM}}$ catheter, and ICE. The FAM was merged with $3 \mathrm{D}$-anatomical chamber reconstructions of LA and PVs derived from pre procedure (up to 24 hours) cardiac computed tomography $(\mathrm{CT})$. Image integration was based on registration involving landmark points and surface alignment using the Cartomerge software (Cartomerge, Biosense Webster., Diamond Bar, CA, USA) as described previously [6].

Circular RF ablation of each vein at the antrum about $1 \mathrm{~cm}$ of veno-atrial junction was performed. The target site for ablation was determined by intracardiac electrogram, CT/FAM integrated image and confirmed by ICE (Figures 1-3). ICE was advanced to right atrium via right or left femoral vein in all patients. It was used for the guidance of trans-septal puncture. Veno-atrial junctions were easily identified by maneuvering the ICE catheter within the right atrium. The $\mathrm{nMARQ}^{\mathrm{TM}}$ catheter was nicely visualized within the LA (Figure 2). Ablation was performed when the location of then MARQ ${ }^{\mathrm{TM}}$ catheter seemed to be optimal at the veno-atrial junctions. We tried to keep good contact of all poles with tissue while keeping all the poles out of veins in order to minimize the risk of PV stenosis. ICE was also used for early detection of acute complication, mainly pericardial effusion and or thrombi in LA.

$\mathrm{RF}$ energy was delivered only in unipolar mode at a maximum power of $15 \mathrm{~W}$ along the posterior wall, and at a maximum powerof $20 \mathrm{~W}$ along the anterior wall at a flow rate of $60 \mathrm{~mL} / \mathrm{min}$. The maximum temperature was set at $43 \mathrm{C}$. Poles with high impedance and or temperature were shut down before or during ablation. One of poles 1 and 10 was shut down if these poles were very close as seen by fluoroscopy or catheter visualization on CT/FAM integrated image and or the presence of artifacts on endocardial signals in order to avoid any energy cross-talk that may causes overheating and thrombus formation. RF ablation was delivered around each vein for two 2 sessions; up to 60 seconds in the first, and up to 40 seconds in the second. The nMARQ ${ }^{\mathrm{TM}}$ catheter was turned clockwise between $90^{\circ}$ and $120^{\circ}$ in the second RF session if it is possible.

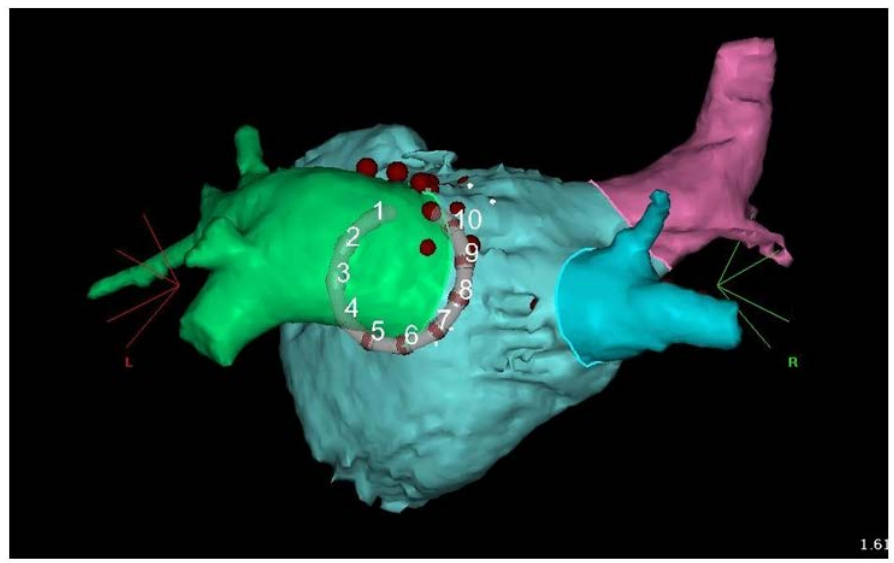

Figure 1. CT image integrated into $3 \mathrm{D}$ mapping system showing 2 separated right pulmonary veins and 1 left common trunk. The nMARQ ${ }^{\mathrm{TM}}$ catheter is positioned at the left common trunk/left atrium junction. This position is confirmed by intracardiac echo. 

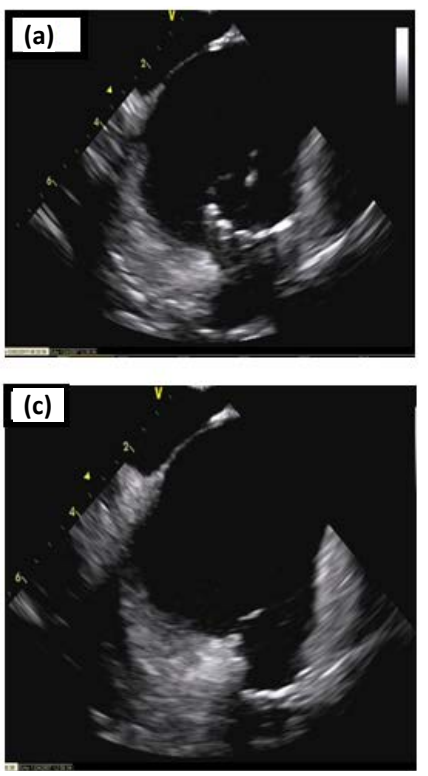

Figure 2. Intracardiac echo showing the position of $\mathrm{nMARQ}^{\mathrm{TM}}$ catheter relative to ostium of left common trunk; (a) The $\mathrm{nMARQ}^{\mathrm{TM}}$ catheter is positioned away from the ostium; (b) The nMARQ ${ }^{\mathrm{TM}}$ catheter is positioned at the ostium; (c) Part of the electrodes of the nMARQ ${ }^{\mathrm{TM}}$ catheter are positioned into the vein; (d) All the electrodes are positioned into the vein.

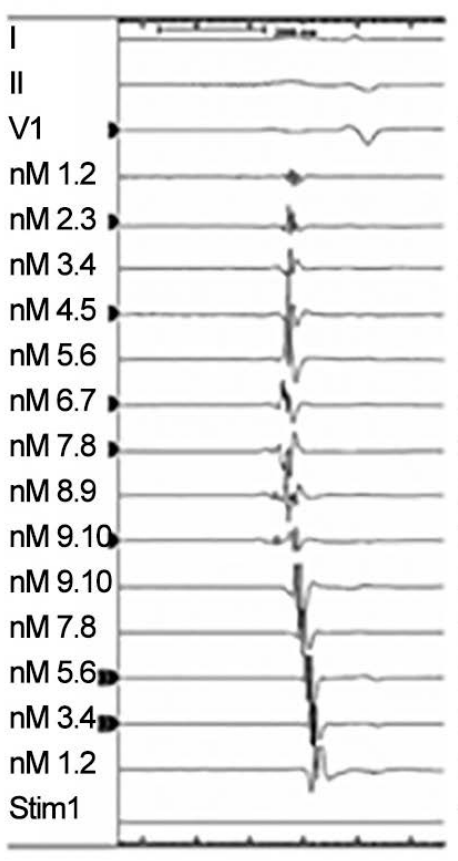

(a)

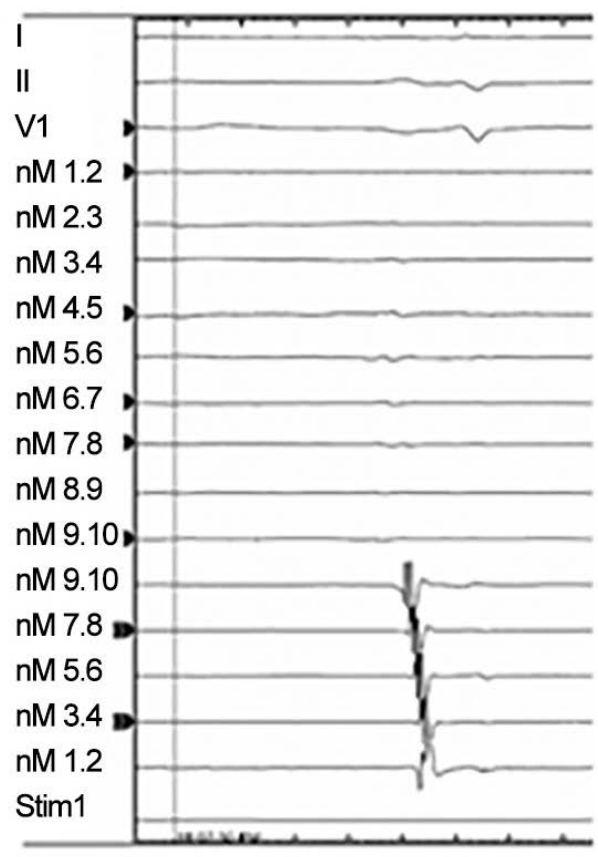

(b)

Figure 3. Surface and nMARQ endocardial recordings of right superior PV. (a) Basal recording before ablation; (b) Recording after ablation and isolation. nM 1,2 up to nM 9,10: nMARQ bipolar endocardial recordings. CS 9, 10 to CS 1, 2: Bipolar recordings from proximal to distal coronary sinus catheter electrodes. 
After that, the nMARQ ${ }^{\mathrm{TM}}$ catheter was advanced beyond the circle of ablation and if possible into the vein after adjusting it to the smallest possible diameter. The position of nMARQ was continually confirmed by ICE. At this point, ablation was delivered via poles showing earliest electrical activity if there is any activity. Lasso catheter (Lasso, Biosense Webster, Diamond Bar, CA, USA) was inserted via the same sheath at the end of ablations if PV isolation was not obvious or PV could not be intubated by the $\mathrm{nMARQ}^{\mathrm{TM}}$ catheter. Repeated ablations were done accordingly after re advancing of the nMARQ $^{\mathrm{TM}}$ catheter. Touch up ablation using standard catheter guided by Lasso was performed if isolation was not achieved after final ablations using the $\mathrm{nMARQ}^{\mathrm{TM}}$ catheter. Lasso was inserted via a second sheath into the same transseptal hole.

All patients were followed in the outpatient clinic 3 months for up to 20 months. Ambulatory 24 hour Holter recording was performed at least once after 3 months of the index procedure. Recurrence was defined as any clinical or documented atrial tachyarrhythmia lasts more 30 seconds after a blanking period of 3 months. All patients were treated with anticoagulation for at least 3 months. Anticoagulation was continued after 3 months in high risk patients according to $\mathrm{CHADS}_{2}$ score. Anti arrhythmic medications were stopped after 3 months if there was no evidence of recurrence.

\section{Statistical Analysis}

Variables are expressed as mean $\pm \mathrm{SD}$. Categorical variables are expressed as numbers and percentages.

\section{Results}

This cohort included 31 patients (mean age $55 \pm 13$ years, 18 males). Baseline characteristics and ablation related data are summarized in Table 1 and Table 2 respectively. Four (13\%) patients had persistent AF and 27 (87\%) had PAF. PVI was achieved in 119 (98\%) out of 121 pulmonary veins identified, and final PVI was obtained in 30 (97\%) out of 31 patients. Lasso catheter was used for PVI confirmation in 16 (52\%) patients. Touch up ablation using standard catheter guided by Lasso catheter was needed in 4 (13\%) patients: left superior and right inferior PVs in 1 patient, superior PVs in 1 patient, inferior PVs in 1 patient, and right PVs in 1 patient. PVs isolation could not achieved in 1 (3\%) patient (left superior and right superior PVs were not isolated) even after touch up ablation. Of note, all the cases that needed touch up ablations were within the first 10 cases. No touch up ablation was needed during the subsequent cases.

Cavotricuspid isthmus (CTI) ablation was performed in the index procedure in 3 (9.7\%) patients because of documented typical atrial flutter using $8 \mathrm{~mm}$ non-irrigated catheter. Roof line was performed in 1 (3\%) patient with persistent AF using the $\mathrm{nMARQ}^{\mathrm{TM}}$ catheter by dragging it from left superior PV to right superior PV.

During follow-up up to 20 months (mean $15.9 \pm 3.6$ months, range 9 - 20 months), 4 (13\%) patients had recurrence of atrial tachyarrhythmia. The recurrence rate would be $13 \%$ if all patients were included in the follow up analysis and would be $14.3 \%$ if 3 
Table 1. Baseline characteristics.

\begin{tabular}{|c|c|}
\hline Parameters & \\
\hline Number of patients & 31 \\
\hline Gender (male) & $18(58 \%)$ \\
\hline Age (years) & $55 \pm 13$ \\
\hline Hypertension & $15(48 \%)$ \\
\hline Diabetes & $3(9.7 \%)$ \\
\hline Ischemic heart disease & $3(9.7 \%)$ \\
\hline \multicolumn{2}{|l|}{$\mathrm{CHADS}_{2}$ score } \\
\hline 0 & $15(48 \%)$ \\
\hline 1 & $12(39 \%)$ \\
\hline$\geq 2$ & $4(13 \%)$ \\
\hline AF duration (months) & $44 \pm 28$ \\
\hline Persistent AF & $4(13 \%)$ \\
\hline \multicolumn{2}{|l|}{ Anti coagulation } \\
\hline Coumadin & $5(16 \%)$ \\
\hline $\mathrm{NOAC}_{\mathrm{s}}$ & $13(42 \%)$ \\
\hline Aspirin & $8(26 \%)$ \\
\hline None & $5(16 \%)$ \\
\hline LVEF (\%) & $58 \pm 6$ \\
\hline \multicolumn{2}{|l|}{ LA size: } \\
\hline Normal & $14(45 \%)$ \\
\hline Mildly enlarged & $13(42 \%)$ \\
\hline Moderately & $4(13 \%)$ \\
\hline
\end{tabular}

$\mathrm{AF}=$ atrial fibrillation; $\mathrm{NOACs}=$ novel oral anticoagulants; $\mathrm{LA}=$ left atrium; $\mathrm{LVEF}=$ left ventricular ejection fraction.

Table 2. Ablation related data.

\begin{tabular}{cc}
\hline Parameters & \\
\hline PVs anatomy & $27(87 \%)$ \\
4 PVs & $4(13 \%)$ \\
Left common trunk & $130 \pm 21$ \\
Procedural time (minutes) & $22 \pm 3$ \\
Total fluoro time (minutes) & $16(52 \%)$ \\
Lasso catheter used & $4(13 \%)$ \\
Touch-up ablation & $119 / 121(98 \%)$ \\
Acute success & $30 / 31(97 \%)$ \\
Isolated PVs & \\
Patients & $9-20$ \\
Follow-up (months) & $15.9 \pm 3.6$ \\
Range & \\
Mean &
\end{tabular}

$\mathrm{AF}=$ atrial fibrillation; $\mathrm{PV}=$ pulmonary vein. 
patients with follow up period less than 12 months were excluded. One patient with paroxysmal AF had early recurrence of AF. He was highly symptomatic despite continuing anti arrhythmic medications. Because of early recurrences after multiple cardioversions, second ablation using $3.5 \mathrm{~mm}$ irrigated catheter (Biosense Webster, Diamond Bar, CA) for point to point ablation and Lasso catheter for PVI confirmation was performed 3 months after the index procedure. Gaps were found at the left superior $\mathrm{PV} /$ left atrial appendage ridge and at the anterior part of right superior PV. After reisolation of PVs, the AF was converted to CTI dependent atrial flutter. This flutter was converted to sinus after ablation of CTI. Another patient with paroxysmal AF had atypical atrial flutter 6 months after the index procedure. During a second procedure, a gap was found at the anterior part of left superior PV. Sinus rhythm was achieved after re-isolation of left superior PV by using $3.5 \mathrm{~mm}$ irrigated catheter (Biosense Webster, Diamond Bar, CA). The other two patients, 1 with persistent AF and 1 with paroxysmal AF had recurrence of AF after 3 and 12 months, respectively. They were treated with anti arrhythmic medications.

Pericardial tamponade occurred in 1 patient 30 minutes after the end of procedure. The patient was treated by emergent pericardiocentesis. Cerebrovascular accident (CVA)/transient ischemic attack (TIA) were not clinically detected in any patient. No clinical complication associated with esophageal thermal injury were observed. Major vascular access injury requiring intervention did not occur in any patient. No late complications were observed in any patient.

\section{Discussion}

PVI using the novel $\mathrm{nMARQ}^{\mathrm{TM}}$ catheter had a high acute success rate and good midterm clinical efficacy during follow-up time up to 20 months with rare major complications. The use of ICE is very helpful for positioning of $\mathrm{nMARQ}^{\mathrm{TM}}$ catheter and confirmation of tissue contact.

The findings in our paper are consistent with recent data published with other groups. However, the workflow of ablation procedure using $\mathrm{nMARQ}^{\mathrm{TM}}$ catheter was not consistent in these reports and the confirmation of PVI was not similar and mostly was not provided with the standard circular multipolar mapping method. In the study of Shin et al. [5], 25 consecutive patients with PAF underwent PVI by using nMARQ ${ }^{\mathrm{TM}}$ catheter. Ablation was guided by left atrial angiogram, fluoroscopy, intracardiac signals from the $\mathrm{nMARQ}^{\mathrm{TM}}$ catheter, and anatomic mapping. Overall, $100 \%$ targeted PVs could be isolated without procedure-related complications. In other study 25 patients with PAF underwent PVI using the $\mathrm{nMARQ}^{\mathrm{TM}}$ catheter [7]. The ablation was guided by electroanatomic map created by $\mathrm{nMARQ}^{\mathrm{TM}}$ and merged with the 3-D magnetic resonance imaging (MRI) shell of LA. PVI, confirmed by Lasso catheter mapping, was achieved in $98 \%$ of PVI identified, and final PVI was obtained in $96 \%$ of patients. Similar results were reported in other study which included 43 patients who underwent PVI using $\mathrm{nMARQ}^{\mathrm{TM}}$. A preablation CT with segmentation of the LA was performed and image integration was used in all procedures [8]. Effective PVI was achieved in $98 \%$ of 
targeted PVs. No clinical procedure-associated complications were noted.

The acute success rate found in this study and reported in recent studies using the novel nMARQ ${ }^{\mathrm{TM}}$ catheter [5] [7] [8] compares with the acute success rate reported by previous studies using a conventional, single-tip approach guided by a $3 \mathrm{D}$ mapping system [9], cryoballoon [3], a visually guided laser balloon catheter [10], or PVAC [4].

We used Lasso catheter (more than half of patients) for confirmation of PVI when the nMARQ ${ }^{\mathrm{TM}}$ catheter could not be advanced into veins or when the isolation is not obvious. The deployment of $\mathrm{nMARQ}^{\mathrm{TM}}$ catheter into right inferior PV can be challenging specially if it is small. Confirmation of PVI was achieved using mainly the $\mathrm{nMARQ}^{\mathrm{TM}}$ catheter in some studies [5] [8], while Lasso catheter was used in others [7]. Shin et al. [5] reported that the deployment of the $\mathrm{nMARQ}^{\mathrm{TM}}$ catheter into PVs for confirmation of PVI was successful in all veins except $28 \%$ of right inferior PVs, thus necessitating the placement of an octopolar diagnostic catheter. In all cases except one left inferior PV, confirmation of PVI was achieved using only the $\mathrm{nMARQ}^{\mathrm{TM}}$ catheter as reported by deneke et al. [8]. However, the concordance between the $\mathrm{nMARQ}^{\mathrm{TM}}$ signals and Lasso signals may not be perfect. Scaglione et al. [7] reported that the overall concordance between Lasso catheter and $\mathrm{nMARQ}^{\mathrm{TM}}$ catheter signals in demonstrating PVI was 78\% [7]. Rosso et al. [11] found that, after RF delivery, the concordance of recordings between the $\mathrm{nMARQ}^{\mathrm{TM}}$ catheter, and the lasso catheter was poor. They concluded that the use of the nMARQ ${ }^{\mathrm{TM}}$ catheter alone may lead to underestimation and overestimation of the number of RF applications required to achieve PV isolation. Importantly, in these studies, the concordance between $\mathrm{nMARQ}^{\mathrm{TM}}$ and Lasso signals was investigated while the $\mathrm{nMARQ}^{\mathrm{TM}}$ catheter positioned around the PV ostium, and the lasso catheter positioned within that vein.

Touch up ablation was needed in 4 patients (PV isolation could not be achieved in 1 patient even after touch up ablation), all of them were within the first 10 cases. It seems that touch up ablation is rarely needed after short learning curve. Touch-up ablation using a 3.5-mm tip open-irrigated catheter was needed only for 1 patient (2 veins) as reported by Scaglione et al. [7] but not in any of the case reported by Shin et al. [5]. One left superior PV and 1 left inferior PV could not be isolated using the $\mathrm{nARQ}^{\mathrm{TM}}$ catheter as reported by Deneke et al. [8]. However, is not mentioned if touch up ablation was performed.

Major clinical complications were not detected in any patient except pericardial tamponade in 1 patient who was treated by pericardiocentesis. No clinical CVA/TIA were detected. We did not perform post ablation MRI, thus silent cerebral lesions could not be ruled out. According to Deneke et al. [8], silent cerebral lesions were found in $33 \%$ of patients detected by post ablation MRI. In contrast, Scaglione et al. [7] reported no silent cerebral ischemia on the basis of MRI after using a workflow to prevent CVA/ TIA that was instituted based on previous experiences. We tried to keep ACT above 320 seconds and to resume anticoagulation 3 hours after removal of sheaths. We were concerned about the exchange of $\mathrm{nMARQ}^{\mathrm{TM}}$ catheter with Lasso catheter when needed for confirmation of PVI. This exchange can increase the risk of thrombus and air emboli. 
However, this exchange seems to be safe if catheters introduction and withdrawal are performed while aspirating from the sheath during their manipulation according to our study and the study of Scaglione et al. [7] who used similar flow chart and did not observed silent cerebral ischemia.

No clinical complication associated with thermal esophageal lesions were observed in our study. Decreasing the power delivered a long the posterior wall (maximum $15 \mathrm{~W}$ in our study) can reduce the risk of esophageal injury. We did not perform esophageal endoscopy, thus could not estimate the rate of non-clinical thermal lesions. Non-clinical thermal esophageal lesions detected by post ablation endoscopy were reported among $33 \%$ of patients in recent study [8]. In this study, ablations were performed with $25 \mathrm{~W}$ over all applicable electrodes in the first 31 patients and with an adapted maximum of $20 \mathrm{~W}$ on the posterior wall of the LA in the last 12 patients in order to reduce the incidence of esophageal thermal lesions. Esophageal lesions were higher (42\% vs. $20 \%$ ) when maximum energy delivery at the posterior wall was $25 \mathrm{~W}$ compared to 20 $\mathrm{W}$, respectively. In the other hand, even all the mentioned studies did not report clinical complication associated with thermal esophageal lesions, fatal outcome like esophagopericardial fistula can occur after ablation of $A F$ using $n M A R Q^{\mathrm{TM}}$ as was previously reported [12].

We used ICE for positioning of $\mathrm{nMARQ}^{\mathrm{TM}}$ catheter at the LA/PVs junctions during creation of FAM allowing precise merging of 3D FAM with 3D CT shell. In addition ICE helps to confirm the position of $\mathrm{nMARQ}^{\mathrm{TM}}$ catheter during ablation and prevents inadvertent ablation within the vein specially if there is an anatomical variants like left common ostium. Furthermore, ICE helps to monitor the contact of the catheter with tissue and to get the optimal position of all the poles while turning the catheter clockwise around the ostium. In addition ICE provides rapid, real-time localization of the esophagus during LA ablation that is comparable to MRI [13]. The importance of ICE for detection of pericardial effusion and tamponade cannot be over emphasized. Other studies used 3D CT or MRI shell merged with electro anatomic mapping or angiogram of LA for positioning of $\mathrm{nMARQ}^{\mathrm{TM}}$ catheter. It is not known if ICE can improve the long term results of PVI using this technology, but we think it can simplify the procedure. It could be possible to perform the procedure without the need for pre procedure CT/MRI or angiogram of LA.

Success rates over $70 \%$ have been reported after point to point ablation of paroxysmal AF ablation and results are maintained during long-term follow-up [14] [15]. Long term follow up is lacking after PVI using $\mathrm{nMARQ}^{\mathrm{TM}}$ catheter. Most of the studies of $\mathrm{nMARQ}^{\mathrm{TM}}$ had short term follow-up time. It was reported that during mean follow-up time of $4.1 \pm 1.6$ months (range 2 - 7 months), no left atrial flutter or tachycardia occurred and after 3 months, sinus rhythm was stable off anti arrhythmic drugs in 17 of $21(80.9 \%)$ patients [5]. Following a 6-month follow-up, 68\% of patients remained free from AF without antiarrhythmic drugs in other study [7]. Zellerhoff et al. [16] reported that single and multiple procedures success rates using $\mathrm{nMARQ}^{\mathrm{TM}}$ during a mean follow-up of $140 \pm 75$ days were $66 \%$ and $77 \%$, respectively. In this study, recurrences of 
AF were mainly due to recovered PV/LA conduction. In a multicenter study, $65 \%$ of paroxysmal AF patients (1-year follow-up data were available in $25 \%$ of patients) and $65 \%$ of persistent AF patients (1-year follow-up data were available in $18 \%$ of patients) were free of arrhythmia at 1 year after $\mathrm{nMARQ}^{\mathrm{TM}}$ ablation for AF [17]. In another multicenter study [18], 180 consecutive patients underwent PV isolation by $\mathrm{nMARQ}^{\mathrm{TM}}$ ablation catheter in 7 centers. In this study $98 \%$ of the targeted veins were isolated (the signals recorded by the $\mathrm{nMARQ}^{\mathrm{TM}}$ catheter were used to demonstrate the PV entrance block). In only 4 patients (2.2\%) a single-point ablation strategy was required to achieve PV isolation. During a mean follow-up of $13.9 \pm 8.2$ months, $27 \%$ of patients with paroxysmal $\mathrm{AF}$ and $30 \%$ of patients with persistent $\mathrm{AF}$ had an atrial arrhythmia relapse. One case of PV stenosis was reported.

Our patients were followed up to 20 months, all of them except 3 patients were followed at least 12 months. The rate of recurrence is acceptable and comparable to other studies, even though the real recurrence rate might be higher if all the patients had a follow-up period of 20 months. The long term efficacy of this novel technology is not known and needed to be addressed with future studies.

\section{Conclusions}

PVI using the novel $\mathrm{nMARQ}^{\mathrm{TM}}$ catheter is safe with good acute and mid-term efficacy.

However, there are some limitations. This study included small number of patients. The patients had different periods of follow up (most of them had at least 12 months follow up). Thus, the actual recurrence rate could be higher. Moreover, the recurrence rate was determined on a clinical basis and on 24 hours holter in some patients, so asymptomatic AF episodes might be missed. Other catheters were used in this study in addition to $\mathrm{nMARQ}^{\mathrm{TM}}$ : Lasso for PVI confirmation in about half of patients and standard 3.5-mm tip open irrigated for touch up ablation to complete PVI in 3 patients. Thus we could not know if the results will be similar if Lasso catheter and or touch up ablation were not used.

We were not aware of clinical PV stenosis. However, the rate of PV stenosis could not be determined in this study because of lack of post ablation imaging. This complication seems to be rare after PVI using the $\mathrm{nMARQ}^{\mathrm{TM}}$ catheter. However, this issue should be addressed by future imaging studies.

Long term follow up and randomized studies are needed to determine the long term efficacy of circular ablation using $\mathrm{nMARQ}^{\mathrm{TM}}$ cathete compared to point to point ablation or to other technologies.

\section{Conflict of Interest}

The authors declare that they have no conflict of interest.

\section{References}

[1] Calkins, H., Kuck, K.H, Cappato, R., Brugada, J., Camm, A.J., Chen, S.A., et al. ( 2012) 2012 HRS/EHRA/ECAS Expert Consensus Statement on Catheter and Surgical Ablation of Atrial 
Fibrillation: Recommendations for Patient Selection, Procedural Techniques, Patient Management and Follow-Up, Definitions, Endpoints, and Research Trial Design: A Report of the Heart Rhythm Society (HRS) Task Force on Catheter and Surgical Ablation of Atrial Fibrillation. Heart Rhythm, 9, 632-696. https://doi.org/10.1016/j.hrthm.2011.12.016

[2] Roten, L., Derval, N., Pascale, P., Scherr, D., Komatsu, Y., Shah, A., et al. (2012) Current Hot Potatoes in Atrial Fibrillation Ablation. Current Cardiology Reviews, 8, 327-346.

https://doi.org/10.2174/157340312803760802

[3] Schmidt, M., Dorwarth, U., Andresen, D., Brachmann, J., Kuck, K.H., Kuniss, M., et al. (2014) Cryoballoon versus RF Ablation in Paroxysmal Atrial Fibrillation: Results from the German Ablation Registry. Journal of Cardiovascular Electrophysiology, 25, 1-7. https://doi.org/10.1111/jce.12267

[4] Deneke, T., de Groot, J.R., Horlitz, M., Mügge, A., Grewe, P.H., et al. (2009) Pulmonary Vein Isolation Using a Novel Decapolar over-the-Wire Mapping and Ablation Catheter. Expert Review of Cardiovascular Therapy, 7, 1341-1347. https://doi.org/10.1586/erc.09.116

[5] Shin, D.I., Kirmanoglou, K., Eickholt, C., Schmidt, J., Clasen, L., Butzbach, B., et al. (2014) Initial Results of Using a Novel Irrigated Multielectrode Mapping and Ablation Catheter for Pulmonary Vein Isolation. Heart Rhythm, 11, 375-383.

https://doi.org/10.1016/j.hrthm.2013.12.008

[6] Kistler, P.M., Earley, M.J., Harris, S., Abrams, D., Ellis, S., Sporton, S.C., et al. (2006) Validation of Three-Dimensional Cardiac Image Integration: Use of Integrated CT Image into Electroanatomic Mapping System to Perform Catheter Ablation of Atrial Fibrillation. Journal of Cardiovascular Electrophysiology, 17, 341-348.

https://doi.org/10.1111/j.1540-8167.2006.00371.x

[7] Scaglione, M., Caponi, D., Anselmino, M., Di Clemente, F., Blandino, A., Ferraris, F., et al. (2014) Pulmonary Vein Isolation with a New Multipolar Irrigated Radiofrequency Ablation Catheter $\left(\mathrm{nMARQ}^{\mathrm{m} \mathrm{m}}\right)$ : Feasibility, Acute and Short-Term Efficacy, Safety, and Impact on Postablation Silent Cerebral Ischemia. Journal of Cardiovascular Electrophysiology, 25, 1299-1305. https://doi.org/10.1111/jce.12500

[8] Deneke, T., Schade, A., Müller, P., Schmitt, R., Christopoulos, G., Krug, J., et al. (2014) Acute Safety and Efficacy of a Novel Multipolar Irrigated Radiofrequency Ablation Catheter for Pulmonary Vein Isolation. Journal of Cardiovascular Electrophysiology, 25, 339-345. https://doi.org/10.1111/jce.12316

[9] Ouyang, F., Bänsch, D., Ernst, S., Schaumann, A., Hachiya, H., Chen, M., et al. (2004) Complete Isolation of Left Atrium Surrounding the Pulmonary Veins: New Insights from the Double-Lasso Technique in Paroxysmal Atrial Fibrillation. Circulation, 110, 2090-2096. https://doi.org/10.1161/01.CIR.0000144459.37455.EE

[10] Dukkipati, S.R., Kuck, K.H., Neuzil, P., Woollett, I., Kautzner, J., McElderry, H.T., et al. (2013) Pulmonary Vein Isolation Using a Visually Guided Laser Balloon Catheter: The First 200-Patient Multicenter Clinical Experience. Circulation: Arrhythmia and Electrophysiolo$g y$, 6, 467-472. https://doi.org/10.1161/CIRCEP.113.000431

[11] Rosso, R., Halkin, A., Michowitz, Y., Belhassen, B., Glick, A. and Viskin, S. (2014) Radiofrequency Ablation of Paroxysmal Atrial Fibrillation with the New Irrigated Multipolar nMARQ Ablation Catheter: Verification of Intracardiac Signals with a Second Circular Mapping Catheter. Heart Rhythm, 11, 559-565. https://doi.org/10.1016/j.hrthm.2013.12.029

[12] Deneke, T., Schade, A., Diegeler, A. and Nentwich, K. (2014) Esophago-Pericardial Fistula Complicating Atrial Fibrillation Ablation Using a Novel Irrigated Radiofrequency Multipolar Ablation Catheter. Journal of Cardiovascular Electrophysiology, 25, 442-443. https://doi.org/10.1111/jce.12308 
[13] Kenigsberg, D.N., Lee, B.P., Grizzard, J.D., Ellenbogen, K.A. and Wood, M.A. (2007) Accuracy of Intracardiac Echocardiography for Assessing the Esophageal Course along the Posterior Left Atrium: A Comparison to Magnetic Resonance Imaging. Journal of Cardiovascular Electrophysiology, 18, 169-173. https://doi.org/10.1111/j.1540-8167.2006.00699.x

[14] Piccini, J.P., Lopes, R.D., Kong, M.H., Hasselblad, V., Jackson, K. and Al-Khatib, S.M. (2009) Pulmonary Vein Isolation for the Maintenance of Sinus Rhythm in Patients with Atrial Fibrillation: A Meta-Analysis of Randomized, Controlled Trials. Circulation: Arrhythmia and Electrophysiology, 2, 626-633. https://doi.org/10.1161/CIRCEP.109.856633

[15] Ouyang, F., Tilz, R., Chun, J., Schmidt, B., Wissner, E., Zerm, T., et al. (2010) Long-Term Results of Catheter Ablation in Paroxysmal Atrial Fibrillation: Lessons from a 5-Year Follow-Up. Circulation, 122, 2368-2377. https://doi.org/10.1161/CIRCULATIONAHA.110.946806

[16] Zellerhoff, S., Daly, M., Lim, H.S., Denis, A., Komatsu, Y., Jesel, L., et al. (2014) Pulmonary Vein Isolation Using a Circular, Open Irrigated Mapping and Ablation Catheter (nMARQ): A Report on Feasibility and Efficacy. Europace, 16, 1296-1303. https://doi.org/10.1093/europace/euu133

[17] Mahida, S., Hooks, D.A., Nentwich, K., Ng, G.A., Grimaldi, M., Shin, D.I., et al. (2015) nMARQ Ablation for Atrial Fibrillation: Results from a Multicenter Study. Journal of Cardiovascular Electrophysiology, 26, 724-729. https://doi.org/10.1111/jce.12698

[18] Stabile, G., de Ruvo, E., Grimaldi, M., Rovaris, G., Soldati, E., Anselmino, M., et al. (2015) Safety and Efficacy of Pulmonary Vein Isolation Using a Circular, Open-Irrigated Mapping and Ablation Catheter: A Multicenter Registry. Heart Rhythm, 12, 1782-1788.

https://doi.org/10.1016/j.hrthm.2015.05.025

\section{Abbreviations}

$\mathrm{AF}=$ atrial fibrillation;

$\mathrm{PV}=$ pulmonary vein;

$\mathrm{RF}=$ radio frequency;

ICE = intracardiac echo;

LA = left atrium;

$\mathrm{CTI}=$ cavotricuspid isthmus;

$\mathrm{CT}=$ computed tomography;

$\mathrm{MRI}=$ magnetic resonance imaging;

CVA = cerebrovascular accident;

TIA $=$ transient ischemic attack. 
Submit or recommend next manuscript to SCIRP and we will provide best service for you:

Accepting pre-submission inquiries through Email, Facebook, LinkedIn, Twitter, etc. A wide selection of journals (inclusive of 9 subjects, more than 200 journals)

Providing 24-hour high-quality service

User-friendly online submission system

Fair and swift peer-review system

Efficient typesetting and proofreading procedure

Display of the result of downloads and visits, as well as the number of cited articles

Maximum dissemination of your research work

Submit your manuscript at: http://papersubmission.scirp.org/

Or contactwjcd@scirp.org 